\title{
Coexistent squamous cell carcinoma and tuberculosis in cervix - A rare occurrence
}

\author{
Raje $\mathrm{P}^{1}$, Bhandari $\mathrm{S}^{2}$ \\ ${ }^{I}$ Department of Surgical Pathology and Cytology, Bombay Hospital, Indore, India. \\ ${ }^{2}$ Department of Pathology, Bombay Hospital, Indore, India.
}

\section{Keywords: \\ Carcinoma; \\ Cervix; \\ Coexistent; \\ Tuberculosis;}

\begin{abstract}
Coexistence of squamous cell carcinoma cervix with tuberculosis cervix, as reported in literature, is a rare entity. Only 8 cases have been reported in the world literature so far. Tuberculosis in general is rampant in developing countries, especially in India, and so is carcinoma cervix and hence one would expect a much higher incidence of coexistence of two. However tuberculosis cervix is a rarely reported disease. We report a case of tuberculosis cervix associated with squamous cell carcinomaand regional lymph node involvement in a postmenopausal woman.
\end{abstract}

\section{INTRODUCTION}

In 1855 Rokitansky propounded that tuberculosis (TB) and malignancy cannot be present in the same organ and a definite antagonism exists between the two. ${ }^{1}$ Morgagni contradicted this view. ${ }^{2}$ Since then existence of both diseases together has been documented in many organs.

Carcinoma cervix is very common and is reported and worked upon extensively. TB in general and of genital organs is also very common, however TB cervix is a rare disease and coexistence of carcinoma cervix with TB is even rarer. Though both diseases are rampant in India, only one case of TB cervix with changes of cervical intraepithelial neoplasia CIN diagnosed cytologically has been reported. ${ }^{3}$

\section{Correspondence:}

Dr. Prakash Raje, MD, DCP

Head, Dept of Surgical Pathology and Cytology

271, Saket, Indore, MP, India, 452018.

Email: drprakashraje@gmail.com
The reported incidence of TB cervix is only $0.1-0.65 \%$ of all tubercular case and $5-24 \%$ of all genital tuberculosis cases. This incidence; quoted in many works also; appear high as in practice one rarely comes across a case of TB cervix.

Diagnostic difficulties occur as typical caseation is not seen, staining for acid fast bacilli (AFB) is negative and isolation of mycobacterium is a cumbersome, time consuming process. In routine practice diagnosing squamous cell carcinoma (SCC) is easier than diagnosing TB and hence it is possible that presence of TB is overlooked with serious consequences to the patient.

\section{CASE REPORT}

A 50 years old postmenopausal woman presented with history of generalized weakness, loss of appetite and weight and bleeding per vaginum since three months. No prior history of significant illness or exposure to 


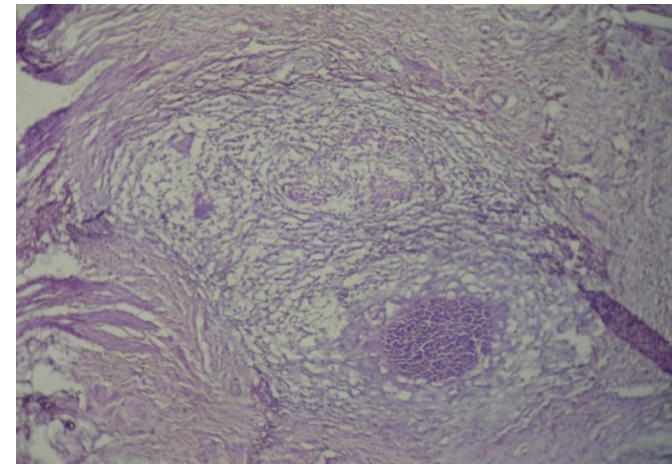

Figure 1: Photomicrograph of cervix: a focus of squamous cell carcinoma along with caseating granulomas, [HE stain, $x$ 400].

TB or immunosuppression. Antibodies to Human immunodeficiency virus were negative.

Ultrasonography showed a hypoechoic mass in the cervix. Other organs were normal. X-ray chest was within normal limits (WNL). Per speculum examination revealed an ulceroproliferative growth that was diagnosed on biopsy as SCC. Wertheim's hysterectomy performed in June 2012 revealed a circumferential growth in cervix, $5 \times 2 \times 0.5 \mathrm{cms}$. Uterine body, tubes and ovaries were normal. Two right and six left pelvic nodes were also resected.

Microscopic examination showed a moderately differentiated keratinising SCC with lymphatic invasion. Multiple granulomas made up of Langhans type of giant cells and epithelioid cells were also seen. (fig.1) There was no caseation in the cervix. Endometrium, rest of uterus, tubes and ovaries did not show any evidence of malignancy or tuberculosis.

One pelvic node showed tumor metastasis. Two nodes showed caseating granulomas. (fig.2) Subsequent urine examination and acetylcholine esterase levels were WNL. Zeihl Neelsen stain was negative both in lymph nodes and in cervix. Culture for mycobacteria was not done. Polymerase Chain Reaction (PCR) for mycobacterium tuberculosis was positive in lymph nodes. No other focus of tuberculosis was found in the body. Amoebiasis and sarcoidosis were ruled out clinically.

A diagnosis of SCC cervix, grade II, with cervical tuberculosis, both involving lymph nodes was made. Diagnosis of tuberculosis in this case was made postoperatively by finding caseating granulomas in lymph node and a positive PCR. Patient was advised chemotherapy (cisplatin and $5 \mathrm{FU}$ ) along with antitubercular treatment. Patient is under close follow up for last 6 months and is doing well. Recent scans are negative for any lymph nodes.

\section{DISCUSSION}

Historically, much before Rokitansky's statement, ${ }^{4}$ Boyle

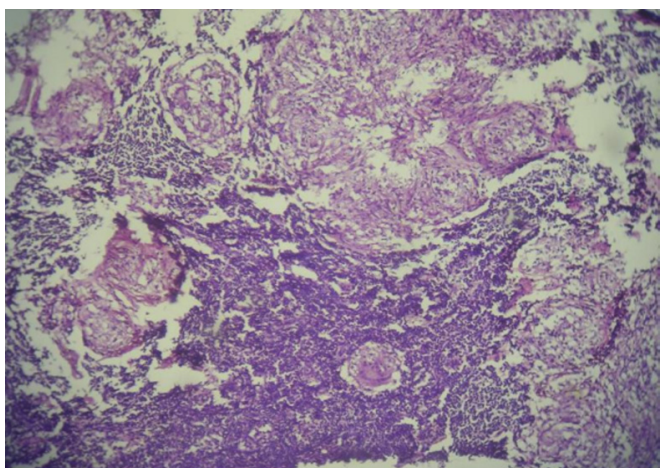

Figure 2: Photomicrograph of lymph node: multiple caseating granuloms effacing the architecture, [HE stain, $x$ 400].

in 1810 had first described the association of TB and malignancy. Since then both have been reported together in practically all the organs in the body. However coexistence of TB with SCC in cervix is a rare occurrence. There are only six cases in literature with coexistent carcinoma and TB in cervix - Wallart et el (1903), Sehottlander et el (1905), Eisenstein et el (1910), Schmidt et el (1914) and Mennin et el (1933). Chien Tien et el in 1985 presented two cases but these cases showed association of generalized upper genital TB with carcinoma cervix.

Mutual influence between TB and cancer is not fully understood. Their togetherness has been explained by many authors in many diverse ways. ${ }^{1}$ It may be that (a) the coexistence is purely coincidental, (b) cancer cachexia may offer good nutritional basis for dormant TB bacilli, and (c) cancer may be superimposed on chronic progressive TB.

Genital TB commonly affects upper genitals in a woman of child bearing age. ${ }^{2}$ Pelvic organs are infected from a primary focus, usually in the chest or abdomen, by haematogenous spread. Cervix is infected either as a part of this process, ${ }^{5-8}$ by lymphatic spread or by direct extension. Primary cervical TB may also occur if a partner suffering from tubercular epididymoorchitis transmits the disease through sexual contact. ${ }^{8}$ Both cancer and TB present with fever, abdominal pain, weight loss, ulceration and growth in the cervix..$^{5-7}$

Gross appearance of TB cervix may be ulcerative with multiple sinuses; hypertrophic with elephantiasis like picture; similar to that of carcinoma - papillary or vegetative growth or a miliary appearance. Diagnosis of cervical TB is usually made by histological examination of biopsy cervix. ${ }^{8,9}$ It is a well-known fact that caseation is rarely present in TB cervix. A third of cases are culture negative. In one study staining for acid fast bacilli was not found to be very useful in making the diagnosis. Isolation of mycobacterium is the gold standard for diagnosis. Molecular probes may be more sensitive than culture but also have reduced specificity.

Histologically diagnosis of SCC, specially with metastasis, is much easier than confirming a diagnosis of $\mathrm{TB}$ in a 
granulomatous lesion. ${ }^{8,9}$

Granulomatous lesions or giant cells in the vicinity of carcinoma in the cervix may be due to amoebiasis, sarcoidosis, foreign body, schistosomiasis or tularemia. ${ }^{1}$ In India only amoebiasis, sarcoidosis and foreign bodies cause granulomatous disease other than tuberculosis.

\section{CONCLUSION}

Since gross appearance and clinical presentation of both these diseases are similar a thorough investigation and identification of two different pathologies in cervix is important for an effective treatment and control of disease. Oversight of TB may lead to over staging of cancer in TNM system; it also alters the postoperative management. Oversight of malignancy may have disastrous outcome for the patient. With resurgence of TB worldwide, there should be a high index of suspicion of TB, in women with an abnormal cervical appearance.

\section{REFERENCES}

1. Lamba H, Byrne M, Goldin R, Jenkins C. Tuberculosis of the cervix: Case presentation and a review of the literature. Sex Transm Inf 2002;78:62-63. Crossref

2. Varma TR. Genital tuberculosis and subsequent fertility. Int $\mathrm{J}$
Gynaecol Obstet 1991;35:1-11. Crossref

3. Bhambani S, Das DK, Singh V, et al. Cervical tuberculosis with carcinoma in situ: a cytodiagnosis. Acta Cytol 1985;29:87-8.

4. Bayle GI. Paris: Gabon; 1810. Recherches sur la phthisie pulmonaire; p. 310 .

5. Chowdhury NNR. Overview of tuberculosis of the female genital tract. J Indian Med Assoc1996;94:345-61. Crossref

6. Sinha R, Gupta D, Tuli N. Genital tract tuberculosis with myometrial involvement. Int J Gynaecol Obstet1997;557:191-2. Crossref

7. Highman WJ. Cervical smears in tuberculous endometritis. Acta Cytol1972;16:16-20. Crossref

8. Sutherland AM, Glen ES, MacFarlane JR. Transmission of genitourinary tuberculosis. Health Bul11982;40:87-91.

9. Shobin D, Sall S, Pellman C. Genitourinary tuberculosis simulating cervical carcinoma. J Reprod Med1976;17:305-8. Crossref 